\title{
On a Subclass of Multivalent Functions with Bounded Positive Real Part
}

\author{
G.Thirupathi \\ Department of Mathematics, Ayya Nadar Janaki Ammal College, Sivakasi-626 124, Tamilnadu, India. \\ gtvenkat79@gmail.com.
}

\begin{abstract}
In the present paper, by introducing a new subclass of multivalent functions with respect to $(j, k)$ - symmetric points, we have obtained the integral representations and conditions for starlikeness using differential subordination.

Keywords: multivalent functions; $(j, k)$ - symmetric points; Differential subordination.
\end{abstract}

\section{Introduction, Definitions And Preliminaries}

Let $\mathscr{H}$ be the class of functions analytic in the open unit disc $\mathbb{U}=\{z \in \mathbb{C}:|z|<1\}$. Let $\mathscr{H}(a, m)$ be the subclass of $\mathscr{H}$ consisting of functions of the form $f(z)=z+a_{m+1} z^{m+1}+a_{m+2} z^{m+2}+\cdots$.

Let $\mathscr{A}_{p}$ be the class of functions $f(z)$, of the form

$f(z)=z^{p}+\sum_{n=p+1}^{\infty} a_{n} z^{n}$

which are analytic in the unit disc $\mathbb{U}=\{z \in \mathbb{C}:|z|<1\}$. And let $\mathscr{A}=\mathscr{A}_{1}$.

We denote by $\mathscr{S}^{*}, \mathscr{C}, \mathscr{K}$ and $\mathscr{C}^{*}$ the familiar subclasses of $\mathscr{A}$ consisting of functions which are respectively starlike, convex, close-to-convex and quasi-convex in $\mathbb{U}$.

Let $\mathscr{S}$ be the subclass of $\mathscr{A}$ consisting of all functions which are univalent in $\mathbb{U}$. Also, let $\mathscr{P}$ denote the class of functions of the form

$$
p(z)=1+\sum_{n=1}^{\infty} c_{n} z^{n}
$$

which are analytic and convex in $\mathbb{U}$ and satisfy the condition

$$
\mathfrak{R}(p(z))>0,(z \in \mathbb{U}) .
$$

Let $f(z)$ and $g(z)$ be analytic in $\mathbb{U}$. Then we say that the function $f(z)$ is subordinate to $g(z)$ in $\mathbb{U}$, if there exists an analytic function $w(z)$ in $\mathbb{U}$ such that $|w(z)|<|z|$ and $f(z)=g(w(z))$, denoted by $f(z) \prec g(z)$. If $g(z)$ is univalent in $\mathbb{U}$, then the subordination is equivalent to $f(0)=g(0)$ and $f(\mathbb{U}) \subset g(\mathbb{U})$.

Motivated by the concept introduced by Sakaguchi in [8], recently several subclasses of analytic functions with respect to $k$-symmetric points were introduced and studied by various authors (see [1], [2], [9], [10] and [12]). Parvatham in ([7]) introduced and investigated $K_{n}(\alpha, h)$ - so called class of $\alpha$ starlike functions with respect to $n$ symmetric points.

Let $k$ be a positive integer and $j=0,1,2, \ldots(k-1)$. A domain $D$ is said to be $(j, k)$-fold symmetric if a rotation of $D$ about the origin through an angle $2 \pi j / k$ carries $D$ onto itself. A function $f \in \mathscr{A}$ is said to be $(j, k)$-symmetrical if for each $z \in \mathbb{U}$

$f(\varepsilon z)=\varepsilon^{j} f(z)$

where $\varepsilon=\exp (2 \pi i / k)$. The family of $(j, k)$-symmetrical functions will be denoted by $\mathscr{F}_{k}^{j}$. For every function $f$ defined on a symmetrical subset $\mathbb{U}$ of $\mathbb{C}$, there exits a unique sequence of $(j, k)$-symmetrical functions $f_{j, k}(z), j=0,1, \ldots, k-1$ such that

$$
f=\sum_{j=0}^{k-1} f_{j, k} .
$$


Also let $f_{j, k}(z)$ be defined by the following equality

$f_{j, k}(z)=\frac{1}{k} \sum_{v=0}^{k-1} \frac{f\left(\varepsilon^{v} z\right)}{\varepsilon^{v p j}}, \quad\left(f \in \mathscr{A}_{p} ; k=1,2, \ldots ; j=0,1,2, \ldots(k-1)\right)$.

where, $v$ is an integer.

This decomposition is a generalization of the well known fact that each function defined on a symmetrical subset $\mathbb{U}$ of $\mathbb{C}$ can be uniquely represented as the sum of an even function and an odd functions (see Theorem 1 of [5]). It is obvious that $f_{j, k}(z)$ is a linear operator from $\mathbb{U}$ into $\mathbb{U}$. The notion of $(j, k)$-symmetrical functions was first introduced and studied by P. Liczberski and J. Polubiński in [5].

The following identities directly follow from (3):

$f_{j, k}\left(\varepsilon^{v} z\right)=\varepsilon^{v p j} f_{j, k}(z)$

$f_{j, k}^{\prime}\left(\varepsilon^{v} z\right)=\varepsilon^{v p j-v} f_{j, k}^{\prime}(z)$

$f_{j, k}^{\prime \prime}\left(\varepsilon^{v} z\right)=\varepsilon^{v p j-2 v} f_{j, k}^{\prime \prime}(z)$

In [4], Karthikeyan et.al., investigated the class

$\mathscr{S}_{j, k}^{p}(b ; \alpha, \beta)=\left\{f \in \mathscr{A}_{p}: \alpha<\operatorname{Re}\left\{1+\frac{1}{b}\left(\frac{z f^{(m+1)}(z)}{f_{j, k}^{(m)}(z)}-p+m\right)\right\}<\beta, 0 \leq \alpha<1<\beta\right\}$.

Motivated by the above concept, in this paper, we introduce and investigate a new subclass of multivalent functions with respect to symmetric points. We now define the following:

Definition 1.1. The function $f \in \mathscr{A}_{p}$ and $\frac{f(z) f^{\prime}(z)}{z} \neq 0$ in $\mathbb{U}$ is said to be in the class $\mathscr{S}_{p}^{j, k}(\gamma ; \lambda, \alpha, \beta)$ of $p$ - valently functions of complex order $\gamma \neq 0$ if and only if it satisfies the condition

$\alpha<\Re\left\{1+\frac{1}{\gamma}\left(\frac{(1-\lambda) z f^{\prime}(z)+\lambda z\left(z f^{\prime}(z)\right)^{\prime}}{(1-\lambda) f_{j, k}(z)+\lambda z f_{j, k}^{\prime}(z)}-p\right)\right\}<\beta, \quad(z \in \mathbb{U})$,

where, $0 \leq \alpha<1<\beta, 0 \leq \lambda \leq 1$ and $f_{j, k}(z) \neq 0$ is defined by the equality (3). Similarly, we say that a function $f \in \mathscr{A}_{p}$ is in the class $\mathscr{C}_{p}^{j, k}(\gamma ; \lambda, \alpha, \beta)$ if and only if

$$
z f^{\prime} \in \mathscr{S}_{p}^{j, k}(\gamma ; \lambda, \alpha, \beta)
$$

Remark 1.1. If $\lambda=0, j=k=p=1$ and $\alpha \geq 0$, then $f(z)$ reduces to the well-known class of starlike functions of complex order. Similarly, if we let $\lambda=1, j=k=p=1$ and $\alpha \geq 0$, then $f(z)$ reduces to the well-known class convex functions of complex order.

We observe that for a given $\alpha$ and $\beta(0 \leq \alpha<1<\beta), f \in \mathscr{S}_{p}^{j, k}(\gamma ; \lambda, \alpha, \beta)$ satisfies each of the following subordination equations

$$
1+\frac{1}{\gamma}\left(\frac{(1-\lambda) z f^{\prime}(z)+\lambda z\left(z f^{\prime}(z)\right)^{\prime}}{(1-\lambda) f_{j, k}(z)+\lambda z f_{j, k}^{\prime}(z)}-p\right) \prec \frac{1+(1-2 \alpha) z}{1-z}
$$

and

$$
1+\frac{1}{\gamma}\left(\frac{(1-\lambda) z f^{\prime}(z)+\lambda z\left(z f^{\prime}(z)\right)^{\prime}}{(1-\lambda) f_{j, k}(z)+\lambda z f_{j, k}^{\prime}(z)}-p\right) \prec \frac{1+(1-2 \beta) z}{1-z} .
$$

Both superordinate functions in the above expressions maps the unit disc onto right half plane, so it is obvious that the above expression is mapped on to a plane having real part greater than $\alpha$ but less than $\beta$.

Kuroki and Owa [3], defined an analytic function $p: \mathbb{U} \rightarrow \mathbb{C}$ by

$p(z)=1+\frac{\beta-\alpha}{\pi} i \log \left(\frac{1-e^{2 \pi i \frac{(1-\alpha)}{(\beta-\alpha)}} z}{1-z}\right)$.

The above function $p$ maps $\mathbb{U}$ onto a convex domain $\Lambda=\{w: \alpha<\operatorname{Re}\{w\}<\beta\}$, conformally. Using this fact and the definition of subordination, we can obtain the following:

Let $f \in \mathscr{A}_{p}$ and $0 \leq \alpha<1<\beta$. Then $f \in \mathscr{S}_{p}^{j, k}(\gamma ; \lambda, \alpha, \beta)$ if and only if

$1+\frac{1}{\gamma}\left(\frac{(1-\lambda) z f^{\prime}(z)+\lambda z\left(z f^{\prime}(z)\right)^{\prime}}{(1-\lambda) f_{j, k}(z)+\lambda z f_{j, k}^{\prime}(z)}-p\right) \prec p(z)$,

and $p(z)$ is of the form

$$
p(z)=1+\sum_{n=1}^{\infty} c_{n} z^{n}
$$

and $c_{n}=\left(\frac{\beta-\alpha}{n \pi}\right) i\left(1-e^{2 n \pi i \frac{(1-\alpha)}{(\beta-\alpha)}}\right)$. 
Lemma 1.1. [6] Let the functions $q$ be univalent in the open unit disc $\mathbb{U}$ and $\theta$ and $\phi$ be analytic in a domain $D$ containing $q(\mathbb{U})$ with $\phi(w) \neq 0$ when $w \in q(\mathbb{U})$. Set $Q(z)=z q^{\prime}(z) \phi(q(z)), h(z)=\theta(q(z))+Q(z)$. Suppose that

1. $Q$ is starlike univalent in $\mathbb{U}$ and

2. $\mathfrak{R}\left(\frac{z h^{\prime}(z)}{Q(z)}\right)>0$ for $z \in \mathbb{U}$.

If

$$
\theta(p(z))+z p^{\prime}(z) \phi(p(z)) \prec \theta(q(z))+z q^{\prime}(z) \phi(q(z)),
$$

then $p(z) \prec q(z)$ and $q$ is the best dominant.

\section{Main Results}

In this section, we prove the integral representation of the function class $\mathscr{S}_{p}^{j, k}(\gamma ; \lambda, \alpha, \beta)$.

Theorem 2.1. Let $f \in \mathscr{S}_{p}^{j, k}(\gamma ; \lambda, \alpha, \beta)$ with $0 \leq \alpha<1<\beta$ and $0<\lambda \leq 1$. Then we have

$f_{j, k}(z)=\frac{1}{\lambda} z^{1-\frac{1}{\lambda}} \int_{0}^{z} \exp \left\{\frac{\gamma}{k} \sum_{v=0}^{k-1} \int_{0}^{u} \frac{1}{\zeta}\left[\frac{\beta-\alpha}{\pi} i \log \left(\frac{1-e^{2 \pi i \frac{(1-\alpha)}{(\beta-\alpha)} w\left(\varepsilon^{v} \zeta\right)}}{1-w\left(\varepsilon^{v} \zeta\right)}\right)\right] d \zeta\right\} u^{\frac{1}{\lambda}+p-2} d u$

where $f_{j, k}(z)$ defined by (3), $w(z)$ is analytic in $\mathbb{U}$ with $w(0)=0$ and $|w(z)|<1$.

Proof. Let $f \in \mathscr{S}_{p}^{j, k}(\gamma ; \lambda, \alpha, \beta)$ with $0 \leq \alpha<1<\beta$ and $0<\lambda \leq 1$. Then we have

$1+\frac{1}{\gamma}\left(\frac{(1-\lambda) z f^{\prime}(z)+\lambda z\left(z f^{\prime}(z)\right)^{\prime}}{(1-\lambda) f_{j, k}(z)+\lambda z f_{j, k}^{\prime}(z)}-p\right)=1+\frac{\beta-\alpha}{\pi} i \log \left(\frac{1-e^{2 \pi i\left(\frac{(1-\alpha)}{(\beta-\alpha)}\right.} w(z)}{1-w(z)}\right)$,

where $w(z)$ is analytic in $\mathbb{U}$ with $w(0)=0$ and $|w(z)|<1$. Substituting $z$ by $\varepsilon^{v} z$ in (7), we have

$1+\frac{1}{\gamma}\left(\frac{(1-\lambda) \varepsilon^{v} z f^{\prime}\left(\varepsilon^{v} z\right)+\lambda \varepsilon^{v} z\left(\varepsilon^{v} z f^{\prime}\left(\varepsilon^{v} z\right)\right)^{\prime}}{(1-\lambda) f_{j, k}\left(\varepsilon^{v} z\right)+\lambda \varepsilon^{v} z f_{j, k}^{\prime}\left(\varepsilon^{v} z\right)}-p\right)=1+\frac{\beta-\alpha}{\pi} i \log \left(\frac{1-e^{2 \pi i\left(\frac{(1-\alpha)}{(\beta-\alpha)}\right.} w\left(\varepsilon^{v} z\right)}{1-w\left(\varepsilon^{v} z\right)}\right)$

Using the identities (4), we have

$1+\frac{1}{\gamma}\left(\frac{(1-\lambda) \varepsilon^{v} z f^{\prime}\left(\varepsilon^{v} z\right)+\lambda \varepsilon^{v} z\left(\varepsilon^{v} z f^{\prime}\left(\varepsilon^{v} z\right)\right)^{\prime}}{(1-\lambda) \varepsilon^{v p j} f_{j, k}(z)+\lambda \varepsilon^{v} z \varepsilon^{v p j-v} f_{j, k}^{\prime}(z)}-p\right)=1+\frac{\beta-\alpha}{\pi} i \log \left(\frac{1-e^{2 \pi i\left(\frac{(1-\alpha)}{(\beta-\alpha)}\right.} w\left(\varepsilon^{v} z\right)}{1-w\left(\varepsilon^{v} z\right)}\right)$.

On simplifying, we get

$\frac{1}{\gamma}\left(\frac{(1-\lambda) \varepsilon^{v-v p j} z f^{\prime}\left(\varepsilon^{v} z\right)+\lambda \varepsilon^{2 v-v p j} z\left(z f^{\prime}\left(\varepsilon^{v} z\right)\right)^{\prime}}{(1-\lambda) f_{j, k}(z)+\lambda z f_{j, k}^{\prime}(z)}-p\right)=\frac{\beta-\alpha}{\pi} i \log \left(\frac{1-e^{2 \pi i\left(\frac{1-\alpha)}{(\beta-\alpha)}\right.} w\left(\varepsilon^{v} z\right)}{1-w\left(\varepsilon^{v} z\right)}\right)$

Let $v=0,1,2, \ldots,(k-1)$ in (10) respectively and summing them, we get

$\frac{1}{\gamma}\left(\frac{(1-\lambda) z f_{j, k}^{\prime}(z)+\lambda z\left(z f_{j, k}^{\prime}(z)\right)^{\prime}}{(1-\lambda) f_{j, k}(z)+\lambda z f_{j, k}^{\prime}(z)}-p\right)=\frac{1}{k} \sum_{v=0}^{k-1} \frac{\beta-\alpha}{\pi} i \log \left(\frac{1-e^{2 \pi i\left(\frac{1-\alpha)}{(\beta-\alpha)} w\left(\varepsilon^{v} z\right)\right.}}{1-w\left(\varepsilon^{v} z\right)}\right)$.

From this equality, we get

$$
\frac{(1-\lambda) f_{j, k}^{\prime}(z)+\lambda\left(z f_{j, k}^{\prime}(z)\right)^{\prime}}{(1-\lambda) f_{j, k}(z)+\lambda z f_{j, k}^{\prime}(z)}-\frac{p}{z}=\frac{\gamma}{k z} \sum_{v=0}^{k-1} \frac{\beta-\alpha}{\pi} i \log \left(\frac{1-e^{2 \pi i\left(\frac{1-\alpha)}{(\beta-\alpha)}\right.} w\left(\varepsilon^{v} z\right)}{1-w\left(\varepsilon^{v} z\right)}\right) .
$$

Integrating, we get

$\log \left(\frac{(1-\lambda) f_{j, k}(z)+\lambda z f_{j, k}^{\prime}(z)}{z^{p}}\right)=\frac{\gamma}{k} \sum_{v=0}^{k-1} \int_{0}^{z} \frac{1}{t}\left(\frac{\beta-\alpha}{\pi} i \log \left(\frac{1-e^{2 \pi i\left(\frac{(1-\alpha)}{(\beta-\alpha)} w\left(\varepsilon^{v} t\right)\right.}}{1-w\left(\varepsilon^{v} t\right)}\right)\right) d t$

Simplifying (13), we have

$(1-\lambda) f_{j, k}(z)+\lambda z f_{j, k}^{\prime}(z)=z^{p} \exp \left\{\frac{\gamma}{k} \sum_{v=0}^{k-1} \int_{0}^{z} \frac{1}{t}\left(\frac{\beta-\alpha}{\pi} i \log \left(\frac{1-e^{2 \pi i \frac{(1-\alpha)}{(\beta-\alpha)}} w\left(\varepsilon^{v} t\right)}{1-w\left(\varepsilon^{v} t\right)}\right)\right) d t\right\}$.

A simple computation in (14), gives the required conclusion of this theorem. 
Theorem 2.2. Let $f \in \mathscr{S}_{p}^{j, k}(\gamma ; \lambda, \alpha, \beta)$ with $0 \leq \alpha<1<\beta$ and $0<\lambda \leq 1$. Then we have

$$
\begin{aligned}
f(z)=\frac{1}{\lambda} z^{1-\frac{1}{\lambda}} \int_{0}^{z} \int_{0}^{u} & \exp \left\{\frac{\gamma}{k} \sum_{v=0}^{k-1} \int_{0}^{\eta} \frac{1}{\zeta}\left[\frac{\beta-\alpha}{\pi} i \log \left(\frac{1-e^{2 \pi i\left(\frac{(1-\alpha)}{(\beta-\alpha)} w\left(\varepsilon^{v} \zeta\right)\right.}}{1-w\left(\varepsilon^{v} \zeta\right)}\right)\right] d \zeta\right\} \\
\times & {\left[p+\frac{\gamma(\beta-\alpha)}{\pi} i \log \left(\frac{1-e^{2 \pi i\left(\frac{1-\alpha)}{(\beta-\alpha)} w(z)\right.}}{1-w(z)}\right)\right] u^{\frac{1}{\lambda}+p-3} d \eta d u }
\end{aligned}
$$

where $w(z)$ is analytic in $\mathbb{U}$ with $w(0)=0$ and $|w(z)|<1$.

Proof. From (7), we have

$$
\begin{aligned}
(1-\lambda) z f^{\prime}(z)+\lambda z\left(z f^{\prime}(z)\right)^{\prime}= & \left((1-\lambda) f_{j, k}(z)+\lambda z f_{j, k}^{\prime}(z)\right) \\
& \times\left[p+\frac{\gamma(\beta-\alpha)}{\pi} i \log \left(\frac{1-e^{2 \pi i\left(\frac{1-\alpha)}{(\beta-\alpha)}\right.} w(z)}{1-w(z)}\right)\right] .
\end{aligned}
$$

From (14) and (16), we have

$$
\begin{aligned}
(1-\lambda) f^{\prime}(z)+\lambda\left(z f^{\prime}(z)\right)^{\prime}= & z^{p-1} \exp \left\{\frac{\gamma}{k} \sum_{v=0}^{k-1} \int_{0}^{z} \frac{1}{t}\left(\frac{\beta-\alpha}{\pi} i \log \left(\frac{1-e^{2 \pi i \frac{(1-\alpha)}{(\beta-\alpha)}} w\left(\varepsilon^{v} t\right)}{1-w\left(\varepsilon^{v} t\right)}\right)\right) d t\right\} \\
& \times\left[p+\frac{\gamma(\beta-\alpha)}{\pi} i \log \left(\frac{1-e^{2 \pi i\left(\frac{1-\alpha)}{(\beta-\alpha)} w(z)\right.}}{1-w(z)}\right)\right] .
\end{aligned}
$$

On simplifying and integrating the above equality (17), we get (15).

If we put $\lambda=1, j=k=1$ in Definition 1.1 and Theorem 2.1, we get the following corollary:

Corollary 2.3. If $f \in \mathscr{A}_{p}$ satisfies the analytic condition

$$
\alpha<\Re\left\{1+\frac{1}{\gamma}\left(1+\frac{z f^{\prime \prime}(z)}{f^{\prime}(z)}-p\right)\right\}<\beta,
$$

then the integral representation of $f(z)$ is given by

$f(z)=\int_{0}^{z} t^{p-1} \exp \left\{\gamma \sum_{v=0}^{k-1} \int_{0}^{t} \frac{1}{\zeta}\left(\frac{\beta-\alpha}{\pi} i \log \left(\frac{1-e^{2 \pi i\left(\frac{1-\alpha)}{(\beta-\alpha)} w\left(\varepsilon^{v} \zeta\right)\right.}}{1-w\left(\varepsilon^{v} \zeta\right)}\right)\right) d \zeta\right\} d t$.

Remark 2.1. If we put $\lambda=1, j=k=1$ in (5) then this result is reduced into the Corollary 2.5 in [4].

Remark 2.2. If we put $\lambda=0, j=k=1$ in (14), then

$$
f(z)=z^{p} \exp \left\{\gamma \sum_{v=0}^{k-1} \int_{0}^{z} \frac{1}{t}\left(\frac{\beta-\alpha}{\pi} i \log \left(\frac{1-e^{2 \pi i\left(\frac{(1-\alpha)}{(\beta-\alpha)} w\left(\varepsilon^{v} t\right)\right.}}{1-w\left(\varepsilon^{v} t\right)}\right)\right) d t\right\} .
$$

Take $p=1$, this result was proved by K.Kuroki and S.Owa [3].

Theorem 2.4. Let the function $h(z)$ analytic in $\mathbb{U}$ be defined by

$$
\begin{aligned}
h(z)=\delta & +(\delta+\kappa) \frac{\beta-\alpha}{\pi} i \log \left(\frac{1-e^{2 \pi i \frac{(1-\alpha)}{(\beta-\alpha)}} z}{1-z}\right)+\kappa\left(\frac{\beta-\alpha}{\pi}\right) i \frac{z\left(1-e^{2 \pi i \frac{(1-\alpha)}{(\beta-\alpha)}}\right)}{(1-z)\left(1-e^{2 \pi i\left(\frac{1-\alpha)}{(\beta-\alpha)} z\right)}\right.} \\
& -\kappa\left(\frac{\beta-\alpha}{\pi}\right)^{2}\left[\log \left(\frac{1-e^{2 \pi i\left(\frac{1-\alpha)}{(\beta-\alpha)}\right.} z}{1-z}\right)\right]^{2}
\end{aligned}
$$

where $\kappa>0, \kappa+\delta>0$. If $f \in \mathscr{A}$ with $\frac{f_{j, k}(z)}{z} \neq 0$ satisfies the condition

$\delta+\frac{(\delta+\kappa)}{\gamma}\left[\frac{F(z)}{F_{j, k}(z)}-1\right]+\frac{\kappa}{\gamma^{2}}\left[\frac{F(z)}{F_{j, k}(z)}-1\right]^{2}+\frac{\kappa}{\gamma}\left[\frac{z F^{\prime}(z)}{F_{j, k}(z)}-\frac{z F(z) F_{j, k}^{\prime}(z)}{\left(F_{j, k}(z)\right)^{2}}\right] \prec h(z)$,

where

$F(z)=(1-\lambda) z f^{\prime}(z)+\lambda z\left(z f^{\prime}(z)\right)^{\prime} \quad$ and $\quad F_{j, k}(z)=(1-\lambda) f_{j, k}(z)+\lambda z f_{j, k}^{\prime}(z)$

then $f \in \mathscr{S}_{1}^{j, k}(\gamma ; \lambda, \alpha, \beta)$. 
Proof. Let the function $p(z)$ be defined by

$p(z)=1+\frac{1}{\gamma}\left(\frac{F(z)}{F_{j, k}(z)}-1\right) \quad(z \in \mathbb{U} ; z \neq 0 ; f \in \mathscr{A})$,

where $p(z)=1+c_{1} z+c_{2} z^{2}+\cdots \in \mathscr{P}, F(z)$ and $F_{j, k}(z)$ defined by (20). On simplification, we get

$z p^{\prime}(z)=\frac{1}{\gamma}\left[\frac{z F^{\prime}(z)}{F_{j, k}(z)}-\frac{z F(z) F_{j, k}^{\prime}(z)}{\left(F_{j, k}(z)\right)^{2}}\right]$.

Thus by (19), we have

$\kappa z p^{\prime}(z)+\kappa p^{2}(z)+(\delta-\kappa) p(z) \prec h(z)$.

Let

$g(z)=1+\frac{\beta-\alpha}{\pi} i \log \left(\frac{1-e^{2 \pi i \frac{(1-\alpha)}{(\beta-\alpha)}} w(z)}{1-w(z)}\right)$.

Set

$$
\theta(w)=\kappa w^{2}+(\delta-\kappa) w \quad \text { and } \quad \phi(w)=\kappa,
$$

it can be easily verified that $\theta$ is analytic in $\mathbb{C}, \phi$ is analytic in $\mathbb{C}$ with $\phi(0) \neq 0$ in the $w$-plane. Also, let

$$
Q(z)=z g^{\prime}(z) \phi(g(z))=\kappa z g^{\prime}(z)
$$

and

$$
h(z)=\theta(g(z))+Q(z)=\kappa(g(z))^{2}+(\delta-\kappa) g(z)+\kappa z g^{\prime}(z) .
$$

Since $g(z)$ is convex univalent in $\mathbb{U}$ provided $\alpha \geq 0$, it gives that $Q(z)$ is starlike univalent in $\mathbb{U}$. In view of the result proved in [3], $g(z)$ given by (23) is starlike for $\alpha \geq 0$, we have

$$
\Re\left(\frac{z h^{\prime}(z)}{Q(z)}\right)=\mathfrak{R}\left\{\kappa\left(\frac{g(z)}{z g^{\prime}(z)}(g(z)-1)+1\right)+\delta \frac{g(z)}{z g^{\prime}(z)}\right\}>0 .
$$

By the application of Lemma 1.1, we get the required assertion of this theorem.

If we put $\lambda=0, \gamma=1$ in Theorem 2.4, we get the following corollary:

Corollary 2.5. Let the function $h(z)$ be defined as in (18). If $f \in \mathscr{A}$ with $\frac{f_{j, k}(z)}{z} \neq 0$ satisfies the condition

$$
\kappa\left\{\frac{z^{2} f^{\prime \prime}(z)}{f_{j, k}(z)}-\frac{z^{2} f^{\prime}(z) f_{j, k}^{\prime}(z)}{\left(f_{j, k}(z)\right)^{2}}+\frac{z^{2}\left(f^{\prime}(z)\right)^{2}}{\left(f_{j, k}(z)\right)^{2}}\right\}+\delta\left(\frac{z f^{\prime}(z)}{f_{j, k}(z)}\right) \prec h(z),
$$

then

$\frac{z f^{\prime}(z)}{f_{j, k}(z)} \prec 1+\frac{\beta-\alpha}{\pi} i \log \left(\frac{1-e^{2 \pi i\left(\frac{1-\alpha)}{(\beta-\alpha)} z\right.}}{1-z}\right)$.

Remark 2.3. If we take $j=k=1$ in the corollary 2.5 , then this result was analogous to the result obtained by Xu et al. in [11].

\section{References}

[1] R. M. Ali, A. O. Badghaish and V. Ravichandran, "Multivalent functions with respect to $n$-ply points and symmetric conjugate points", Comput. Math. Appl., 60, no. 11, (2010), 2926-2935

[2] R. Chandrashekar, Rosihan M Ali, S. K. Lee, V. Ravichandran, "Convolutions of meromorphic multivalent functions with respect to $n$-ply points and symmetric conjugate points", Appl. Math. Comput. 218, no. 3, (2011), 723-728.

[3] K. Kuroki and S. Owa, "Notes on new class for certain analytic functions", RIMS Kokyuroku, 1772 (2011) pp. 2125.

[4] K. R. Karthikeyan, K. Srinivasan and K. Ramachandran, "On A Class Of Multivalent Starlike Functions With A Bounded Positive Real Part", Palestine Journal of Mathematics, Vol. 5(1) (2016), 59-64.

[5] P. Liczberski and J. Połubiński, "On ( $j, k)$-symmetrical functions", Math. Bohem., 120, no. 1, (1995), 13-28.

[6] S. S. Miller and P. T. Mocanu, "Subordinants of differential superordinations", Complex Var. Theory Appl., 48, no. 10 (2003), 815-826.

[7] R. Parvatham, S. Radha, "On $\alpha$-starlike and $\alpha$-close-to-convex functions with respect to $n$-symmetric points", Indian J. Pure Appl. Math., 17, no. 9 (1986), 1114-1122.

[8] K. Sakaguchi, "On a certain univalent mapping", J. Math. Soc. Japan, 11 (1959), 72-75.

[9] Z. G. Wang, C. Y. Gao and S. M. Yuan, "On certain subclasses of close-to-convex and quasi-convex functions with respect to $k$-symmetric points", $J$. Math. Anal. Appl., 322, no. 1, (2006), 97-106.

[10] Z. G. Wang, Y.P.Jiang and H.M.Srivastava, "Some subclasses of multivalent analytic functions involving the Dziok-Srivastava operator", Integral Transforms Spec. Funct., 19, no. 1-2 (2008), 129-146.

[11] N. Xu and D. Yang, "Some criteria for starlikeness and strongly starlikeness", Bull. Korean Math. Soc., 42, no. 3 (2005), 579-590.

[12] S. M. Yuan and Z. M. Liu, "Some properties of $\alpha$-convex and $\alpha$-quasiconvex functions with respect to $n$-symmetric points", Appl. Math. Comput., 188, no. 2,(2007), 1142-1150. 